\title{
ANALYTICAL SOLUTIONS FOR THE FREE SURFACE HYDROSTATIC EULER EQUATIONS*
}

\author{
A.C. BOULANGER ${ }^{\dagger}$, M.-O. BRISTEAU ${ }^{\ddagger}$, AND J. SAINTE-MARIE ${ }^{\S}$
}

\begin{abstract}
In this paper we propose a large set of analytical solutions (FRESH-ASSESS) for the hydrostatic incompressible Euler system in $2 \mathrm{~d}$ and $3 \mathrm{~d}$. These solutions mainly concern free surface flows but partially free surface flows are also considered. These analytical solutions can be especially useful for the validation of numerical schemes.
\end{abstract}

Key words. Incompressible Euler equations, analytical solutions, free surface flows, entropic shocks.

AMS subject classifications. 35Q30, 35Q35, 76D05.

\section{Introduction}

Models arising in fluid dynamics often based on the Navier-Stokes equations are generally difficult to analyze both at the mathematical or numerical level. As a consequence the derivation of efficient numerical schemes and their validations are complex. A way to circumvent this difficulty is to have access to analytical solutions of the considered problem and to compare the simulated solutions with them. Even if this approach is not a complete validation of the numerical schemes, it enables one to have a good knowledge of the behavior of the discrete schemes in front of typical and complex situations particularly when the family of analytical solutions is large enough. Moreover it makes possible the study of the convergence order of a simulated solution towards the reference one.

Starting from the incompressible Navier-Stokes equations, much work has been done in the derivation of relatively simple mathematical models for shallow water flows and long non-linear water waves. For these models, analytical solutions are often available. Thus the Saint-Venant system [3], which is well suited for the modeling of river hydraulics, has been widely studied by many authors. In a chronological order, the most famous contributions are: analytical solutions for the dam break situation by Ritter [17], the nonlinear wave propagation in shallow water open channels by Stoker [18], solutions of the Saint-Venant system for a flow respectively over a ridge and in a parabolic bowl by Houghton [9] and Thacker [19]. MacDonald et al. [14, 15] have proposed a wide family of analytical solutions with bottom friction, hydraulic jumps, and various open-channel cross-sections. Likewise for the Korteweg-de Vries equations [10] and related systems - generally corresponding to potential flows [13] such as the Boussinesq system [5], and the Green-Naghdi model [7], various analytical solutions of soliton type are available $[11,12,4]$.

*Received: June 30, 2012; accepted (in revised form): October 31, 2012. Communicated by Francois Bouchut.

†UPMC Univ Paris 06, CNRS UMR 7598, Laboratoire Jacques-Louis Lions, F-75005, Paris and INRIA Rocquencourt, B.P. 105, F-78153 Le Chesnay Cedex, France and CETMEF, 134 rue de Beauvais - CS 60039, 60280 Margny les Compiègne, France (Anne-Céline.Boulanger@inria.fr).

${ }^{\ddagger}$ CETMEF, 134 rue de Beauvais - CS 60039, 60280 Margny les Compiègne and INRIA Rocquencourt, B.P. 105, F-78153 Le Chesnay Cedex, France and UPMC Univ Paris 06, CNRS UMR 7598, Laboratoire Jacques-Louis Lions, F-75005, Paris, France (Marie-Odile.Bristeau@inria.fr).

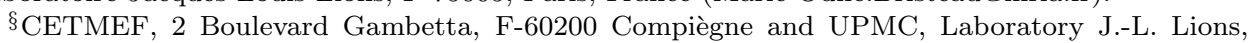
CNRS UMR 7598, F-75005, Paris and INRIA Rocquencourt, B.P. 105, F-78153 Le Chesnay Cedex, France (Jacques.Sainte-Marie@inria.fr). 
Unfortunately, the previously mentioned models correspond to averaged (along a spatial coordinate) or irrotational approximations of the Navier-Stokes equations. In this paper we propose various analytical solutions for the incompressible hydrostatic Euler equations in the following situations: stationary flows, transient flows, free (or partially free) surface, external forcing, simplified viscosity terms. These solutions are denoted hereafter by the acronym FRESH-ASSESS corresponding to FREe Surface Hydrodynamics Analytical SolutionS for Euler SystemS. The analytical solutions are available in $2 \mathrm{~d}$ and $3 \mathrm{~d}$ for a large class of bottom geometry. Some of these solutions admit entropic shocks that are characterized.

The main concern of the authors is to exhibit reference solutions that can be used for the validation of numerical codes. In the case of a regular flow, the solution is analytic. For nonsmooth solutions, e.g. with shocks, the solution is obtained via the resolution of a simple ODE. If the proposed analytical solutions correspond to physical phenomena, they do not correspond to useful situations for practical use of the models. The analytical solutions are obtained as in MacDonald et al. [14, 15]; starting from a given water depth and an admissible velocity field, we derive the corresponding topography profile.

The paper is organized as follows. Starting from the incompressible and hydrostatic Euler equations (Section 2), we present in Section 3 the 2d FRESH-ASSESS solutions in various configurations. The $3 \mathrm{~d}$ extension of these solutions is given in Section 4. In Section 5, the various analytical solutions are illustrated in typical situations.

\section{The Euler equations}

We start from the $3 \mathrm{~d}$ hydrostatic and incompressible free surface Euler equations $[6,8]$ with gravity:

$$
\begin{aligned}
\frac{\partial u}{\partial x}+\frac{\partial v}{\partial y}+\frac{\partial w}{\partial z} & =0, \\
\frac{\partial u}{\partial t}+u \frac{\partial u}{\partial x}+v \frac{\partial u}{\partial y}+w \frac{\partial u}{\partial z}+\frac{\partial p}{\partial x} & =0, \\
\frac{\partial v}{\partial t}+u \frac{\partial v}{\partial x}+v \frac{\partial v}{\partial y}+w \frac{\partial v}{\partial z}+\frac{\partial p}{\partial y} & =0, \\
\frac{\partial p}{\partial z} & =-g,
\end{aligned}
$$

where the $z$ axis represents the vertical direction. We consider this system for

$$
t>0, \quad(x, y) \in \Omega, \quad z_{b}(x, y) \leq z \leq \eta(x, y, t),
$$

where $\Omega$ is a bounded domain of $\mathbb{R}^{2}, \eta(x, y, t)$ represents the free surface elevation, and $\mathbf{u}=(u, v, w)^{T}$ is the velocity vector. The gravity acceleration is denoted $g$ and $p$ is the fluid pressure. The bottom geometry is $z_{b}$ so the water depth is $H=\eta-z_{b}$; see figure 2.1. Equation (2.1) represents the incompressibility (mass conservation equation) and equations (2.2), (2.3) are respectively the momentum equilibria of forces along the $x$ and $y$ axes. Because of the hydrostatic assumption, the momentum equilibrium of forces along the vertical axis reduces to (2.4).

The system (2.1)-(2.4) is completed with boundary conditions. Classically, at the free surface we have the kinematic boundary condition

$$
\frac{\partial \eta}{\partial t}+u_{s} \frac{\partial \eta}{\partial x}+v_{s} \frac{\partial \eta}{\partial y}-w_{s}=0
$$




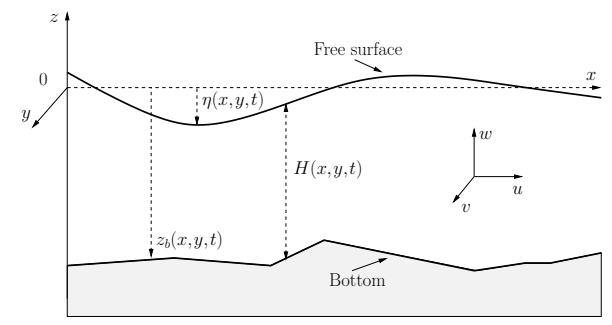

FIG. 2.1. Notations: water height $H(x, y, t)$, free surface $\eta(x, y, t)$, and bottom $z_{b}(x, y)$.

where the subscript $s$ denotes the value of the considered quantity at the free surface. The dynamical condition at the free surface takes into account the equilibrium with the atmospheric pressure, and we have

$$
p_{s}=p^{a}(x, y, t),
$$

where $p^{a}=p^{a}(x, y, t)$ is a given function corresponding to the atmospheric pressure. At the bottom, the kinematic boundary condition corresponds to a no-penetration condition

$$
u_{b} \frac{\partial z_{b}}{\partial x}+v_{b} \frac{\partial z_{b}}{\partial y}-w_{b}=0
$$

where the subscript $b$ denotes the value of the considered quantity at the bottom.

The boundary condition (2.6) coupled with (2.4) gives

$$
p=p^{a}+g(\eta-z),
$$

so the hydrostatic Euler system (2.1)-(2.4) can be written under the form

$$
\begin{aligned}
& \frac{\partial H}{\partial t}+\frac{\partial}{\partial x} \int_{z_{b}}^{\eta} u d z+\frac{\partial}{\partial y} \int_{z_{b}}^{\eta} v d z=0, \\
& w=-\frac{\partial}{\partial x} \int_{z_{b}}^{z} u d z-\frac{\partial}{\partial y} \int_{z_{b}}^{z} v d z, \\
& \frac{\partial u}{\partial t}+u \frac{\partial u}{\partial x}+v \frac{\partial u}{\partial y}+w \frac{\partial u}{\partial z}+g \frac{\partial \eta}{\partial x}=-\frac{\partial p^{a}}{\partial x}, \\
& \frac{\partial v}{\partial t}+u \frac{\partial u}{\partial x}+v \frac{\partial v}{\partial y}+w \frac{\partial u}{\partial z}+g \frac{\partial \eta}{\partial y}=-\frac{\partial p^{a}}{\partial y} .
\end{aligned}
$$

Equation (2.8) results from an integration of the divergence free condition (2.1), along $z$ from $z_{b}$ to $\eta$, coupled with the kinematic relation (2.5). Similarly equation (2.9) results from an integration of the divergence free condition from $z_{b}$ to $z$, where the boundary condition (2.7) is used. Notice that (2.8) and (2.9) are equivalent to (2.1), (2.5), and (2.7).

For smooth solutions, the hydrostatic Euler system (2.1)-(2.7)—or equivalently (2.8)-(2.11) - is completed with an energy equality, namely

$$
\begin{aligned}
\frac{\partial}{\partial t} \int_{z_{b}}^{\eta}\left(E+p^{a}\right) d z+ & \frac{\partial}{\partial x} \int_{z_{b}}^{\eta} u(E+g(\eta-z)+g z) d z \\
& +\frac{\partial}{\partial y} \int_{z_{b}}^{\eta} u(E+g(\eta-z)+g z) d z=H \frac{\partial p^{a}}{\partial t}
\end{aligned}
$$


with

$$
E=\frac{u^{2}}{2}+\frac{v^{2}}{2}+g z
$$

\section{2d analytical solutions}

For the sake of simplicity, we first restrict our presentation to $2 \mathrm{~d}$ analytical solutions. The considered horizontal domain is $\Omega=\left[0, L_{x}\right]$. The $3 \mathrm{~d}$ extension of the proposed solutions is given in Section 4.

In this section, we first propose stationary solutions for the system (2.1)-(2.4). The regularity of the solutions is examined in paragraphs 3.3 and 3.4. Then we exhibit analytical solutions for the Euler system in the following situations: external forcing, viscosity terms, reactive tracer, transient solutions, or a partially free surface.

3.1. Main idea. We first consider the system (2.8)-(2.11) where all the variables are independent of $y$. Stationary solutions of this system must fulfill

$$
\begin{aligned}
& \frac{\partial}{\partial x} \int_{z_{b}}^{\eta} u d z=0 \\
& w=-\frac{\partial}{\partial x} \int_{z_{b}}^{z} u d z \\
& u \frac{\partial u}{\partial x}+w \frac{\partial u}{\partial z}+g \frac{\partial \eta}{\partial x}=-\frac{\partial p^{a}}{\partial x} .
\end{aligned}
$$

Except in paragraphs 3.7.1 and 3.8.1, we assume in this section $p^{a}=C s t$.

From (3.3), it follows that

$$
u \frac{\partial u}{\partial x}+w \frac{\partial u}{\partial z}
$$

must be independent of $z$, and this is the most difficult requirement to satisfy. The underlying idea for the derivation of the analytical solutions is the following: to satisfy (3.1), we seek for $u$ under the form

$$
u=\alpha \frac{\varphi^{\prime}\left(z-z_{b}\right)}{\varphi(H)}
$$

with $\varphi(0)=0, \alpha=C s t$. Then from $(3.2), w$ is given by

$$
w=-\alpha \frac{\partial}{\partial x} \frac{\varphi\left(z-z_{b}\right)}{\varphi(H)}
$$

and replacing the previous expressions for $u$ and $w$ in (3.3) implies that (3.4) is independent of $z$ when

$$
\left(\varphi^{\prime}\left(z-z_{b}\right)\right)^{2}-\varphi\left(z-z_{b}\right) \varphi^{\prime \prime}\left(z-z_{b}\right)=C s t
$$

which is ensured for

$$
\varphi(z)=\sin (z)
$$




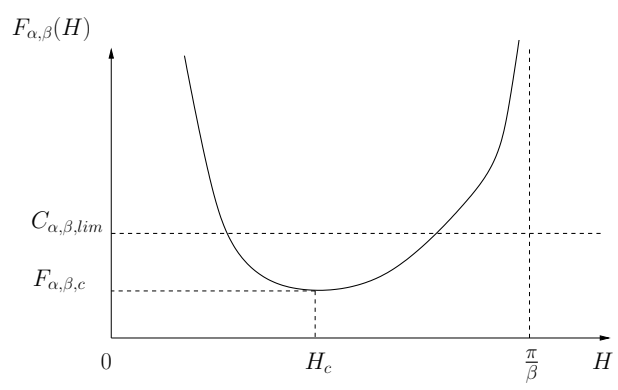

FIG. 3.1. Function $H \mapsto F_{\alpha, \beta}(H)$.

3.2. General continuous stationary solutions. The following proposition gives smooth analytical solutions for the system (3.1)-(3.3).

Proposition 3.1. If $\alpha, \beta$, and $\bar{z}_{b}$ are three real constants and $H_{0}$ a non-negative real $C^{1}(\Omega)$ function such that $\sin \left(\beta H_{0}(x)\right) \neq 0$ for all $x \in \Omega$, then $u=u_{\alpha, \beta}, w=w_{\alpha, \beta}$, and $z_{b}$ respectively given by

$$
\begin{aligned}
z_{b} & =\bar{z}_{b}-H_{0}-\frac{\alpha^{2} \beta^{2}}{2 g \sin ^{2}\left(\beta H_{0}\right)}, \\
u_{\alpha, \beta} & =\frac{\alpha \beta}{\sin \left(\beta H_{0}\right)} \cos \left(\beta\left(z-z_{b}\right)\right), \\
w_{\alpha, \beta} & =\alpha \beta\left(\frac{\partial z_{b}}{\partial x} \frac{\cos \left(\beta\left(z-z_{b}\right)\right)}{\sin \left(\beta H_{0}\right)}+\frac{\partial H_{0}}{\partial x} \frac{\sin \left(\beta\left(z-z_{b}\right)\right) \cos \left(\beta H_{0}\right)}{\sin ^{2}(\beta H)}\right),
\end{aligned}
$$

satisfy the 2d stationary Euler equations (3.1)-(3.3) with $\eta=H_{0}+z_{b}$.

Proof. Following (3.5), (3.7), we propose to choose $u$ under the form

$$
u=u_{\alpha, \beta}=\frac{\alpha \beta}{\sin (\beta H)} \cos \left(\beta\left(z-z_{b}\right)\right)
$$

and from (3.6), $w$ is given by

$$
w=w_{\alpha, \beta}=\alpha \beta\left(\frac{\partial z_{b}}{\partial x} \frac{\cos \left(\beta\left(z-z_{b}\right)\right)}{\sin (\beta H)}+\frac{\partial H}{\partial x} \frac{\sin \left(\beta\left(z-z_{b}\right)\right) \cos (\beta H)}{\sin ^{2}(\beta H)}\right),
$$

then equation $(3.3)$ reduces to

$$
\frac{\partial}{\partial x}\left(\frac{\alpha^{2} \beta^{2}}{2 \sin ^{2}(\beta H)}+g\left(H+z_{b}\right)\right)=0
$$

and the definition (3.8) for $z_{b}$ with $H=H_{0}$ completes the proof.

The previous proposition is only valid for smooth solutions. The regularity and uniqueness of the solutions and their derivation are discussed in the two following paragraphs. 
3.3. Continuity of the solutions. Here we adopt a slightly different strategy, considering we have a given topography $z_{b}(x), x \in \Omega=\left[0, L_{x}\right]$, given values for $\alpha>0$ and $\beta$, and a boundary value for $H$, namely, $H_{\text {lim }}=H(0)$ or $H_{l i m}=H\left(L_{x}\right)$. $H(x)$ will be given by the solution of a simple ODE. We assume $u$ satisfies (3.11), $w$ satisfies (3.12), and it remains to solve (3.13) to obtain $H$.

We introduce the function

$$
F_{\alpha, \beta}(x): x \mapsto g x+\frac{\alpha^{2} \beta^{2}}{2 \sin ^{2}(\beta x)},
$$

and we point out that $F_{\alpha, \beta}$ is not one-to-one (see figure 3.1). When considering smooth solutions - and with the notations of figure 3.1 - the resolution of (3.13) is equivalent to find for all $x \in \Omega$ a solution to

$$
F_{\alpha, \beta}(H(x))+g z_{b}(x)=F_{\alpha, \beta}\left(H_{l i m}\right)+g z_{b, l i m} .
$$

If the bottom profile is enough regular, equation (3.13) can be written under the form

$$
\left(g-\alpha^{3} \beta^{2} \frac{\cos (\beta H)}{\sin ^{3}(\beta H)}\right) \frac{\partial H}{\partial x}=-g \frac{\partial z_{b}}{\partial x},
$$

This previous equation remains well defined until the quantity

$$
\left(g-\alpha^{3} \beta^{2} \frac{\cos (\beta H)}{\sin ^{3}(\beta H)}\right)
$$

vanishes to zero and the critical water depth $H_{c}$ satisfies

$$
\frac{\cos \left(\beta H_{c}\right)}{\sin ^{3}\left(\beta H_{c}\right)}=\frac{g}{\alpha^{3} \beta^{2}} .
$$

In other words, if (3.14) admits a solution in $H_{c}$, then it admits a solution for all $x \in \Omega$.

So, we have now to study the function

$$
f: x \rightarrow \frac{\cos (\beta x)}{\sin ^{3}(\beta x)},
$$

which is defined over $\mathbb{R}-\{n \pi / \beta\}, n \in \mathbb{Z}$, periodic with period $\pi / \beta$. It is a nonincreasing function over each interval $\left.I_{\beta, n}=\right] n \pi / \beta,(n+1) \pi / \beta[$. We can then conclude that for each interval $I_{\beta, n}$, there exists only one solution for equation (3.16). Moreover, since the roots of $f$ are easily defined as $(n+1 / 2) \pi / \beta$, we can conclude from the fact that $\frac{g}{\alpha^{3} \beta^{2}}$ is positive that the solution of equation (3.16) is in the interval ]$n \pi / \beta,(n+1 / 2) \pi / \beta[$. In the following we will consider $n=0$.

Using the notations of figure 3.1, we easily find that the solution $H_{c}$ of equation (3.16) satisfies

$$
H_{c}=\frac{\arcsin r_{c}^{2}}{\beta} \text { and } \quad F_{\alpha, \beta, c}=g H_{c}+\frac{\alpha^{2} \beta^{2}}{2 r_{c}^{4}},
$$

where $r_{c}^{2}$ is the unique real solution of the polynomial equation

$$
P(X)=g^{2} X^{3}+\alpha^{4} \beta^{6} X-\alpha^{4} \beta^{6}=0,
$$


with

$$
r_{c}=\sqrt{\frac{\sqrt[3]{12 \alpha^{4}} \beta^{2}}{6 g} \frac{-\sqrt[3]{12 \alpha^{4}} \beta^{2}+\left(\sqrt[3]{9 g+\sqrt{12 \alpha^{4} \beta^{6}+81 g^{2}}}\right)^{2}}{\sqrt[3]{9 g+\sqrt{12 \alpha^{4} \beta^{6}+81 g^{2}}}}} .
$$

Since we have $P(0) \leq 0$ and $P(1) \geq 0$, we remark that $0 \leq r_{c} \leq 1$.

The previous results allow to write the following proposition.

Proposition 3.2. If for any $x \in \Omega$, the problem

$$
F_{\alpha, \beta}(H(x))=F_{\alpha, \beta}\left(H_{l i m}\right)+g\left(z_{b, l i m}-z_{b}(x)\right)=C_{\alpha, \beta, l i m}-g z_{b}(x) \geq 0
$$

has a solution $H(x) \geq 0$, then $\left(H, u_{\alpha, \beta}, w_{\alpha, \beta}\right)$ are solutions of (3.1)-(3.3). This result can also be expressed in the following way: if the problem (3.19) admits a solution $\mathrm{H}_{c}$ in $x=x_{c}$, or equivalently if

$$
F_{\alpha, \beta, c} \leq C_{\alpha, \beta, l i m},
$$

then it admits a solution for all $x \in \Omega$. And it is easy to prove that if $z_{b} \in C^{k}(\Omega)$, $k \geq 0$, then $\left(H, u_{\alpha, \beta}\right) \in C^{k}(\Omega)$ and $w_{\alpha, \beta} \in C^{k-1}(\Omega)$.

Let $\Omega_{c}$ be the subset of $\Omega$ containing the points $x$ for which the problem (3.19) has no solution. We are now interested in situations where $\Omega_{c} \neq \emptyset$.

3.4. Discontinuous solutions. Let us consider now discontinuous solutions. We define the operator $[A]=A^{+}-A^{-}$, where the superscripts ${ }^{+}$and ${ }^{-}$define the variables right after and before the discontinuity.

Stationary shocks of the system (2.12), (3.1), and (3.3) are characterized by

$$
\begin{array}{r}
{\left[\int_{z_{b}}^{\eta} u d z\right]=0} \\
{\left[\int_{x_{0}}^{x} \int_{z_{b}}^{\eta}\left(u \frac{\partial u}{\partial x}+w \frac{\partial u}{\partial z}+g \frac{\partial H}{\partial x}\right) d z\right]=0} \\
{\left[\int_{z b}^{\eta}\left(\frac{u^{3}}{2}+g \eta u\right) d z\right]=\mathcal{D} \leq 0}
\end{array}
$$

which reduce to

$$
\begin{array}{r}
{\left[\frac{g}{2} H^{2}+\frac{\alpha^{2} \beta^{2} H}{2 \sin (\beta H)^{2}}+\frac{\alpha^{2} \beta}{2} \frac{\cos (\beta H)}{\sin (\beta H)}\right]=0,} \\
{\left[\frac{\alpha^{3} \beta^{2}}{2 \sin ^{2}(\beta H)}+g \alpha\left(H+z_{b}\right)\right]=\mathcal{D} \leq 0,}
\end{array}
$$

since $u=u_{\alpha, \beta}$.

If $\Omega_{c}=\Omega$, then the parameters $\alpha, \beta$, and the boundary condition $H_{l i m}$ are not compatible, so we assume $\Omega_{c} \neq \Omega$. Without loss of generality we assume $H_{\text {lim }}=H\left(L_{x}\right)$ and $\alpha \geq 0$. We also suppose here $z_{b} \in C^{1}(\Omega)$ with a unique $\left.x_{c} \in \stackrel{\circ}{\Omega}=\right] 0, L_{x}$ [ such that

$$
z_{b, c}=\max _{x \in \Omega} z_{b}(x)
$$




$$
\left.\frac{\partial z_{b}}{\partial x}\right|_{x=x_{c}}=0
$$

If $\Omega_{c} \neq \emptyset$ then the problem (3.19) has no solution, at least for $x=x_{c} \in \Omega_{c}$, and the solution will be discontinuous. From (3.23), for $x=x_{c}$ the solution $H$ of

$$
\left(g-\frac{\alpha^{2} \beta^{3} \cos (\beta H)}{\sin (\beta H)^{3}}\right) \frac{\partial H}{\partial x}=0,
$$

and the condition

$$
\left.\frac{\partial H}{\partial x}\right|_{x=x_{c}}=0, \quad \text { with } \quad g-\frac{\alpha^{2} \beta^{3} \cos (\beta H)}{\sin (\beta H)^{3}} \neq 0,
$$

would give that a continuous solution exists over $\Omega$, which contradicts the assumption that $x_{c} \in \Omega_{c}$. So $H_{c}=H\left(x_{c}\right)$ necessarily satisfies (3.16), which is equivalent to (3.18) and is given by $(3.17)$. Over the interval $\left[0, x_{c}\right]$, the solution will be continuous and hence

$$
\left[\frac{\alpha^{3} \beta^{2}}{2 \sin ^{2}(\beta H)}+g \alpha\left(H+z_{b}\right)\right]_{x=0}^{x=x_{c}}=0 .
$$

Over the interval $\left[x_{c}, L_{x}\right]$, we have

$$
\left[\frac{\alpha^{3} \beta^{2}}{2 \sin ^{2}(\beta H)}+g \alpha\left(H+z_{b}\right)\right]_{x=L_{x}}^{x=x_{c}}=\alpha\left(F_{\alpha, \beta, c}+g z_{b, c}-F_{\alpha, \beta}\left(H_{l i m}\right)-g z_{b, l i m}\right),
$$

and by construction $F_{\alpha, \beta, c}+g z_{b, c} \leq C_{\alpha, \beta, l i m}$, so we have $\mathcal{D} \leq 0$ in (3.21).

If a shock occurs, its abscissa $x_{s}$ and the associated water depth are characterized by (3.20)-(3.21), which correspond to

$$
\begin{aligned}
& {\left[\frac{g}{2} H^{2}+\frac{\alpha^{2} \beta^{2} H}{2 \sin ^{2}(\beta H)}+\frac{\alpha^{2} \beta}{2} \frac{\cos (\beta H)}{\sin (\beta H)}\right]_{H_{s, l}}^{H_{s, r}}=0,} \\
& \frac{\alpha^{2} \beta^{2}}{2 \sin ^{2}\left(\beta H_{c}\right)}+g\left(H_{c}+z_{b, c}\right)=\frac{\alpha^{2} \beta^{2}}{2 \sin ^{2}\left(\beta H_{s, l}\right)}+g\left(H_{s, l}+z_{b, s}\right), \\
& \frac{\alpha^{2} \beta^{2}}{2 \sin ^{2}\left(\beta H_{l i m}\right)}+g\left(H_{l i m}+z_{b, l i m}\right)=\frac{\alpha^{2} \beta^{2}}{2 \sin ^{2}\left(\beta H_{s, r}\right)}+g\left(H_{s, r}+z_{b, s}\right),
\end{aligned}
$$

where $z_{b, s}$ is the bottom topography at the abscissa of the shock and $H_{s, l}, H_{s, r}$ are the water depth at the left and right of the shock. If the solutions of the system (3.24)-(3.26) are continuous, this means the outflow boundary condition cannot be satisfied. In this case, the torrential regime takes place from $x=x_{c}$ to $x=L_{x}$.

In practice, the solution $H(x)$ is obtained as follows. Starting from the value $H_{\text {lim }}$ at abscissa $x_{\text {lim }}$, we calculate the solution of the algebraic equation (3.14) or equivalently the ODE (3.15). If no singularity appears, the problem is solved. If for one abscissa equation (3.20) cannot be solved, then $x_{c}$ must be determined by (3.22), (3.23). Then (3.24)-(3.26) allows one to build the whole solution over $\Omega$.

An illustration of the analytical solutions obtained in the situations with and without shocks is given in paragraphs 5.1 and 5.2. 
3.5. Solutions with external forcing. We consider the same hydrostatic Euler system with free surface as previously, but we add an external forcing and a viscosity term (in the vertical direction). The governing equations of the considered system are

$$
\begin{aligned}
\frac{\partial}{\partial x} \int_{z_{b}}^{\eta} u d z & =0, \\
\frac{\partial u}{\partial x}+\frac{\partial w}{\partial z} & =0, \\
u \frac{\partial u}{\partial x}+w \frac{\partial u}{\partial z}+g \frac{\partial \eta}{\partial x} & =f_{x}+\nu \frac{\partial^{2} u}{\partial z^{2}},
\end{aligned}
$$

where $f_{x}$ is a volumetric source term and the atmospheric pressure $p^{a}$ is supposed to be constant. The following proposition holds.

Proposition 3.3. If we choose $f_{x}=\beta \nu u$ and $\beta \geq 0$-which corresponds to an energy supply for the system - then for any bottom profile $z_{b}$ of the form (3.8), the quantities $u=u_{\alpha, \beta}, w=w_{\alpha, \beta}$ given by (3.9) and (3.10) with $H=H_{0}$ are solutions of (3.27)(3.29).

Even if the situations encountered in this paragraph - with an equilibrium between the external forcing and the viscosity - are different from those of the preceding paragraph, the resulting water depth and velocity fields are similar and we do not present any illustration for this situation.

3.6. Coupling with a reactive tracer. We consider in this paragraph the stationary hydrostatic Euler system governed by (3.1)-(3.2) with $p^{a}=C s t$ and coupled with an advection-reaction type equation of the form

$$
u \frac{\partial T}{\partial x}+w \frac{\partial T}{\partial z}=\frac{T_{0}-T}{\tau} .
$$

The equation (3.30) models for example the transport and the reaction of a tracer.

In order to build solutions for the system (3.1)-(3.2) and (3.30) we use the analytical solutions proposed in paragraph 3.2. Thus, since the only remaining constraint for the choice of functions $T, T_{0}$, and $\tau$ is to satisfy (3.30), there exists many solutions for this problem. We present hereafter two distinct choices.

Let $x \mapsto H_{0}(x)$ be a continuous, non negative function defined on $\Omega$, and we choose the bottom topography $z_{b}$ under the form defined by (3.8).

3.6.1. First choice. Suppose $T_{0}=T_{0}(x)$ and $\tau=\tau(x)$ are defined by

$$
\tau=\frac{\sin \left(\beta H_{0}(x)\right)^{2}}{\alpha \beta^{2} \cos \left(b H_{0}(x) \frac{\partial H_{0}}{\partial x}\right.} \quad \text { and } \quad T_{0}=\alpha b \frac{\gamma\left(z-z_{b}(x)\right)}{\sin \left(b H_{0}(x)\right)},
$$

with $\gamma=\gamma(x)=\frac{1}{\cot (x)-\csc (x)}$. Then the velocity fields $u_{\alpha, \beta}, w_{\alpha, \beta}$ defined by (3.9) and (3.10) for $H=H_{0}$, and $T$ defined by

$$
T(x, z)=-\gamma\left(z-z_{b}(x)\right) u_{\alpha, \beta}(x, z),
$$

are solutions of (3.1)-(3.2) coupled with (3.30). 
3.6.2. Second choice. Similarly, the velocity fields $u_{\alpha, \beta}, w_{\alpha, \beta}$ defined by (3.9) and (3.10) for $H=H_{0}$, and $T$ defined by

$$
T(x, z)=e^{-\left(H(x)-\left(z-z_{b}(x)\right)\right)},
$$

with $T_{0}=0$ and

$$
\frac{1}{\tau}=\alpha \beta \frac{\cos \left(\beta\left(z-z_{b}\right)\right)}{\sin (\beta H)}\left(1-\frac{\tan \left(\beta\left(z-z_{b}\right)\right)}{\tan (\beta H)}\right) \frac{\partial H}{\partial x},
$$

are solutions of (3.1)-(3.2) coupled with (3.30).

3.7. Transient analytical solutions. The stationary solutions presented in the preceding paragraphs allow us to validate spatial discretization techniques for the considered equations. In order to evaluate the accuracy of time schemes, transient solutions are necessary. In this paragraph we give two examples of time dependent analytical solutions.

3.7.1. First situation. We now consider the hydrostatic Navier-Stokes system defined by

$$
\begin{aligned}
& \frac{\partial}{\partial x} \int_{z_{b}}^{\eta} u d z=0, \\
& w=-\frac{\partial}{\partial x} \int_{z_{b}}^{z} u d z \\
& \frac{\partial u}{\partial t}+u \frac{\partial u}{\partial x}+w \frac{\partial u}{\partial z}+g \frac{\partial \eta}{\partial x}=-\frac{\partial p^{a}}{\partial x}+\nu \frac{\partial^{2} u}{\partial z^{2}},
\end{aligned}
$$

where $p=p^{a}$ is the atmospheric pressure at the free surface. We assume the velocity fields $u, w$ can vary with respect to time $t$ whereas the water depth $H=H(x)$ only depends on the spatial coordinate $x$.

As in paragraph 3.2 , let $x \mapsto H_{0}(x)$ be a continuous, nonnegative function defined on $\Omega$, choose the bottom topography $z_{b}$ under the form (3.8), and the external pressure $p^{a}$ is given by

$$
p^{a}=-\frac{\alpha^{2} \beta^{2}}{2 \sin ^{2}\left(\beta H_{0}\right)}\left(1-e^{-\nu \beta^{2} t}\right) .
$$

Then the velocity fields

$$
\begin{gathered}
\tilde{u}_{\alpha, \beta}=\frac{\alpha \beta}{\sin \left(\beta H_{0}\right)} \cos \left(\beta\left(z-z_{b}\right)\right) e^{-\nu \beta^{2} t}, \\
\tilde{w}_{\alpha, \beta}=\frac{\alpha \beta e^{-\nu \beta^{2} t}}{\sin ^{2}\left(\beta H_{0}(x)\right)}\left(\sin \left(\beta\left(z-z_{b}(x)\right)\right) \cos \left(\beta H_{0}(x)\right) \frac{\partial H_{0}}{\partial x}\right. \\
\left.\quad+\cos \left(\beta\left(z-z_{b}(x)\right)\right) \frac{\partial z_{b}}{\partial x} \sin \left(\beta H_{0}(x)\right)\right),
\end{gathered}
$$

and the water depth $H_{0}$ satisfy the equations (3.31)-(3.33).

This situation is very similar to what has been proposed in paragraph 3.2 ; the contribution of the horizontal and vertical velocities decreasing exponentially is replaced by the effects of the external pressure $p^{a}$. These analytical solutions are not illustrated.

The system (3.31)-(3.33) can admit discontinuous solutions, but this would imply a discontinuous atmospheric pressure $p^{a}$ that has no physical meaning. 
3.7.2. Analytical solution with a travelling wave. In this paragraph, we consider that the bottom topography $z_{b}=z_{b}(x, t)$ can vary with respect to time and $p^{a}=C s t$. Let us consider the system

$$
\begin{aligned}
& \frac{\partial H}{\partial t}+\frac{\partial}{\partial x} \int_{z_{b}}^{\eta} u d z=0, \\
& w=-\frac{\partial}{\partial x} \int_{z_{b}}^{z} u d z, \\
& \frac{\partial u}{\partial t}+u \frac{\partial u}{\partial x}+w \frac{\partial u}{\partial z}+g \frac{\partial \eta}{\partial x}=f_{x},
\end{aligned}
$$

with $f_{x}$ given by

$$
f_{x}=-\frac{\partial z_{b}}{\partial t} \frac{\partial u}{\partial z} .
$$

The energy balance associated with this system is

$$
\frac{\partial}{\partial t} \int_{z_{b}}^{\eta} E d z+\frac{\partial}{\partial x} \int_{z_{b}}^{\eta} u(E+g(\eta-z)+g z) d z=-\frac{1}{2} \frac{\partial z_{b}}{\partial t}\left(u_{s}^{2}-u_{b}^{2}\right),
$$

with $E=\frac{u^{2}}{2}+g z$.

If $H_{0}(x)$ is a nonnegative quantity such that the bottom topography is given by (3.8), then $H(x, t)=H_{0}\left(x-c_{0} t\right), u_{\alpha, \beta}(x, z, t)$ given by

$$
u_{\alpha, \beta}(x, z, t)=c_{0}+\frac{\alpha \beta}{\sin (\beta H)} \cos \left(\beta\left(z-z_{b}\right)\right),
$$

and $w$ obtained from (3.10) are solutions of (3.34)-(3.36).

3.8. Interactions with structures. We are not only interested in situations of free surface flows, so we consider now situations without free surface or where only a part of the water surface is free.

3.8.1. 2d analytical solutions with partially free surface. Such a situation can be obtained when the atmospheric pressure term $p^{a}$ depends on the flow characteristics $H, u$, and $w$. This corresponds to the case where the flow interacts with a lid. In such a situation, the closure of the system is ensured with a lid displacement law of the form

$$
p^{a}=K(x)\left|\frac{\sqrt{\eta}-\sqrt{\eta_{m}}}{\sqrt{\eta_{m}}}\right|_{+},
$$

with $\eta=H+z_{b}, \eta_{m}=\eta_{m}(x)$ a given function, and $K($.$) a real nonnegative function.$ Such an expression for $p^{a}$ mimics the interaction between a deformable lid and the free surface. For more information about the origins and the mechanical justifications of formula (3.37), the reader can refer to [16].

Thus, if the water depth $H$ satisfies the ODE

$$
\frac{\partial}{\partial x}\left(\frac{\alpha^{2} \beta^{2}}{2 \sin ^{2}(\beta H)}+g H-K(x)\left|\frac{\sqrt{\eta}-\sqrt{\eta_{m}}}{\sqrt{\eta_{m}}}\right| \mathbf{1}_{H+z_{b} \geq \eta_{m}}\right)=-g \frac{\partial z_{b}}{\partial x},
$$


then $H$ and $u_{\alpha, \beta}, w_{\alpha, \beta}$ given by (3.9) and (3.10) are solutions of the considered problem. In particular, if $H_{0}(x)$ is any nonnegative function then $\bar{H}=\bar{\eta}-z_{b}$, with $z_{b}$ given by (3.8) and

$$
\bar{\eta}=\frac{K(x)\left(H_{0}+z_{b}\right)}{\left(\frac{\alpha^{2} \beta^{2}}{2 \sin ^{2}\left(\beta H_{0}\right)}+g\left(H_{0}+z_{b}\right)+K\right)^{2}},
$$

satisfies (3.38).

3.8.2. Analytical solutions in cylindrical coordinates. We now consider the cylindrical representation of the incompressible Euler equations for an axisymmetric flow with the assumption of no tangential velocity. The gravity is also neglected. We denote $u_{x}$ and $u_{r}$ the velocities along the $x$ axis and radial coordinate respectively. The continuity equation reads

$$
\frac{1}{r} \frac{\partial r u_{r}}{\partial r}+\frac{\partial u_{x}}{\partial x}=0
$$

and the momentum equations reduces to

$$
\begin{aligned}
u_{r} \frac{\partial u_{x}}{\partial r}+u_{x} \frac{\partial u_{x}}{\partial x}+\frac{\partial p}{\partial x} & =0 \\
\frac{\partial p}{\partial r} & =0
\end{aligned}
$$

Suppose the flow takes place in a deformable pipe parallel to the $x$ axis and of radius $R(x)$. The kinematic boundary condition at $r=R(x)$ ensuring the fluid does not leave the pipe is given by

$$
\frac{\partial R}{\partial t}+\left.\frac{\partial R}{\partial x} u_{x}\right|_{r=R}-\left.u_{r}\right|_{r=R}=0
$$

Equation (3.41) gives $p=p(x)$ and as in paragraph 3.8.1, the system (3.39)-(3.42) is closed with a wall displacement law of the form

$$
p(x)=K(x)\left(R(x)^{\gamma}-R_{m}(x)^{\gamma}\right),
$$

where $K($.$) and R_{m}($.$) are two nonnegative given functions and \gamma$ is a positive constant.

Using (3.39) and (3.42), the mass conservation in the pipe reads as

$$
\int_{0}^{R(x)} r u_{x} d r=C s t
$$

and the radial velocity $u_{r}$ is given by

$$
u_{r}=\frac{1}{r} \int_{0}^{r} r u_{x} d r
$$

Let us consider the velocity field $u_{x}$ given by

$$
u_{x}=\alpha \beta \frac{\cosh \left(\beta r^{2}\right)}{\sinh \left(\beta R(x)^{2}\right)},
$$

so that all solutions $R(x)$ of

$$
\frac{\partial}{\partial x}\left(\frac{\alpha^{2} \beta^{2}}{2 \sinh ^{2}\left(\beta R(x)^{2}\right)}+K(x)\left(R(x)^{\gamma}-R_{m}(x)^{\gamma}\right)\right)=0
$$


are solutions of the system (3.39)-(3.44). In particular if $R_{0}(x)$ is any nonnegative function then $\bar{R}(x)$ defined by

$$
\bar{R}(x)=\left(\frac{\alpha^{2} \beta^{2}}{2 K(x) \sinh ^{2}\left(\beta R_{0}(x)^{2}\right)}+R_{0}(x)^{\gamma}\right)^{\frac{1}{\gamma}},
$$

is a solution. It must be noticed that, whatever the choices for the positive functions $K(x)$ and $R_{m}(x)$, all of the solutions of (3.45) are necessarily continuous and no shocks can occur.

An illustration of the situations proposed here is given in paragraph 5.3.1.

\section{Analytical solutions in $\mathbf{3 d}$}

In this paragraph, we propose a $3 \mathrm{~d}$ extension of the analytical solutions obtained in paragraph 3.2.

From (2.8)-(2.11), stationary solutions of the 3d Euler system with free surface must fulfill

$$
\begin{aligned}
& \frac{\partial}{\partial x} \int_{z_{b}}^{\eta} u d z+\frac{\partial}{\partial y} \int_{z_{b}}^{\eta} v d z=0 \\
& u \frac{\partial u}{\partial x}+v \frac{\partial u}{\partial y}+w \frac{\partial u}{\partial z}+g \frac{\partial \eta}{\partial x}=\frac{\partial p^{a}}{\partial x}, \\
& u \frac{\partial v}{\partial x}+v \frac{\partial v}{\partial y}+w \frac{\partial v}{\partial z}+g \frac{\partial \eta}{\partial y}=\frac{\partial p^{a}}{\partial y}, \\
& w=-\frac{\partial}{\partial x} \int_{z_{b}}^{z} u d z-\frac{\partial}{\partial y} \int_{z_{b}}^{z} v d z
\end{aligned}
$$

where we have considered $p^{a}=0$. Let us introduce the velocity fields

$$
\begin{aligned}
& u_{\alpha_{1}, \beta}=\frac{\alpha_{1} \beta}{\sin (\beta H)} \cos \left(\beta\left(z-z_{b}\right)\right), \\
& v_{\alpha_{2}, \beta}=\frac{\alpha_{2} \beta}{\sin (\beta H)} \cos \left(\beta\left(z-z_{b}\right)\right),
\end{aligned}
$$

so that we clearly have

$$
\int_{z_{b}}^{\eta} u_{\alpha_{1}, \beta} d z=\alpha_{1} \quad \text { and } \quad \int_{z_{b}}^{\eta} v_{\alpha_{2}, \beta} d z=\alpha_{2} .
$$

Using (4.4), we obtain

$$
\begin{gathered}
w_{\alpha_{1}, \alpha_{2}, \beta}=\frac{\beta}{\sin ^{2}(\beta H)}\left(\sin \left(\beta\left(z-z_{b}\right)\right) \cos (\beta H)\left(\alpha_{1} \frac{\partial H}{\partial x}+\alpha_{2} \frac{\partial H}{\partial y}\right)\right. \\
\left.+\cos \left(\beta\left(z-z_{b}\right)\right) \sin (\beta H)\left(\alpha_{1} \frac{\partial z_{b}}{\partial x}+\alpha_{2} \frac{\partial z_{b}}{\partial y}\right)\right),
\end{gathered}
$$

and the momentum equations along $x$ and $y$ (4.2)-(4.3) reduce to

$$
\begin{aligned}
& g \frac{\partial \eta}{\partial x}-\alpha_{1} \beta^{3} \frac{\cos (\beta H)}{\sin ^{3}(\beta H)}\left(\alpha_{1} \frac{\partial H}{\partial x}+\alpha_{2} \frac{\partial H}{\partial y}\right)=0 \\
& g \frac{\partial \eta}{\partial y}-\alpha_{2} \beta^{3} \frac{\cos (\beta H)}{\sin ^{3}(\beta H)}\left(\alpha_{1} \frac{\partial H}{\partial x}+\alpha_{2} \frac{\partial H}{\partial y}\right)=0
\end{aligned}
$$


or equivalently

$$
\begin{aligned}
& \frac{\partial}{\partial x}\left(g H+\frac{\alpha_{1}^{2} \beta^{2}}{2 \sin ^{2}(\beta H)}\right)+\frac{\partial}{\partial y}\left(\frac{\alpha_{1} \alpha_{2} \beta^{2}}{2 \sin ^{2}(\beta H)}\right)=-g \frac{\partial z_{b}}{\partial x} \\
& \frac{\partial}{\partial x}\left(\frac{\alpha_{1} \alpha_{2} \beta^{2}}{2 \sin ^{2}(\beta H)}\right)+\frac{\partial}{\partial y}\left(g H+\frac{\alpha_{2}^{2} \beta^{2}}{2 \sin ^{2}(\beta H)}\right)=-g \frac{\partial z_{b}}{\partial y}
\end{aligned}
$$

where $H$ is the unknown. Therefore a necessary condition for the system (4.8)-(4.9) to admit a solution is that the water depth $H$ is of the form

$$
H(x, y)=H_{0}\left(\alpha_{1} x+\alpha_{2} y\right) \quad \text { or } \quad H(x, y)=H_{0}\left(\alpha_{2} x-\alpha_{1} y\right)
$$

Only the second choice leads to nontrivial solutions, and thus for any nonnegative function $H_{0}(x)$, the bottom topography given by

$$
z_{b}(x, y)=\bar{z}_{b}-H_{0}\left(\alpha_{1} x+\alpha_{2} y\right)-\frac{\left(\alpha_{1}^{2}+\alpha_{2}^{2}\right) \beta^{2}}{2 g \sin ^{2}\left(\beta H_{0}\left(\alpha_{1} x+\alpha_{2} y\right)\right)},
$$

and the velocity fields $u, v$, and $w$ respectively given by (4.5), (4.6), and (4.7) are solutions of (4.1)-(4.4). These $3 \mathrm{~d}$ analytical solutions are illustrated in paragraph 5.4.

\section{Illustrative examples}

For some of the models investigated above, illustrations of the exhibited analytical solutions are given. In the following, except for paragraphs 5.3 .2 and 5.4, we always consider the situation of a $L_{x}=20$ meter-long basin with constant width and vertical shores. Since the initial and boundary conditions do not depend on the variable $y$ and the bottom geometry satisfies $z_{b}=z_{b}(x)$, it is a $2 \mathrm{~d}(x-z)$ problem.

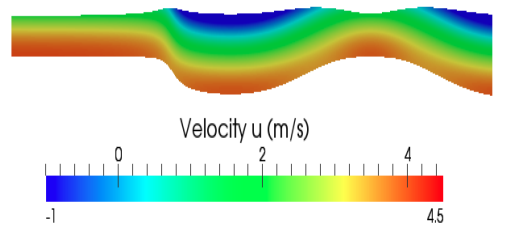

(a)

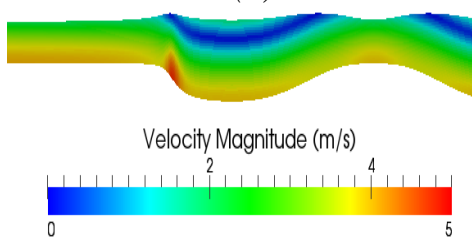

(c)

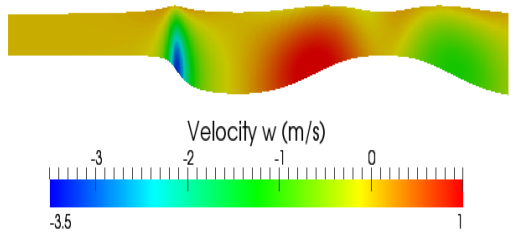

(b)

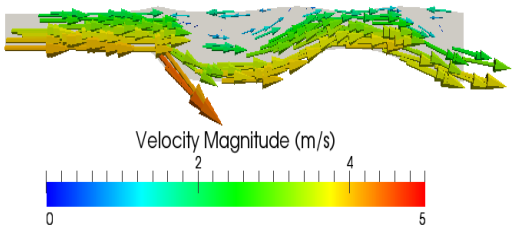

(d)

FIG. 5.1. Surface level of the continuous solution and (a) horizontal velocity, (b) vertical velocity, (c) velocity norm, (d) velocity field (arrow).

5.1. Stationary continuous solutions. In this paragraph we present typical analytical solutions for the problem examined in paragraph 3.2. Let us consider the bottom geometry $z_{b}(x)$ defined by (3.8), where $H_{0}(x)$ is given by

$$
H_{0}(x)=\frac{1}{2}+\frac{\pi}{2}+\frac{\alpha}{10} \tan ^{-1}\left(\frac{N}{L_{x}}\left(x-\frac{L_{x}}{3}\right)\right)-e^{-\frac{N}{L_{x}^{2}}\left(x-\frac{3}{4 L_{x}}\right)^{2}},
$$




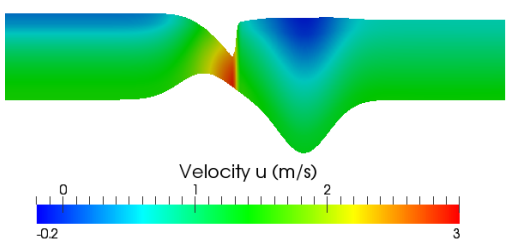

(a)

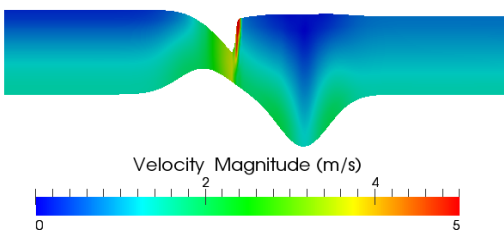

(c)

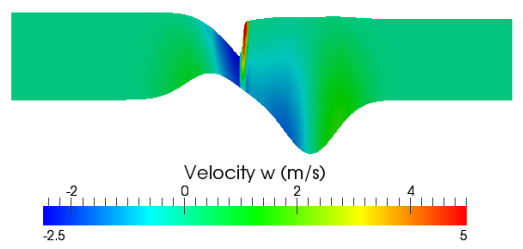

(b)

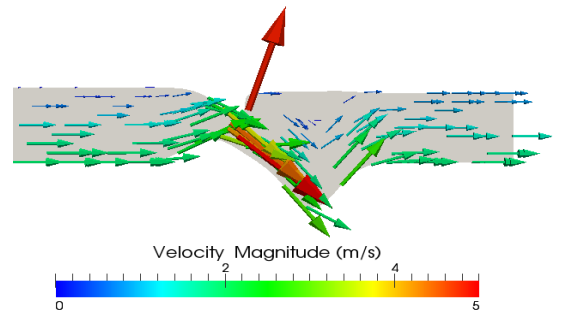

(d)

FIG. 5.2. Surface level of the solution with shock and (a) horizontal velocity, (b) vertical velocity, and (c) velocity norm, (d) velocity field (arrow).

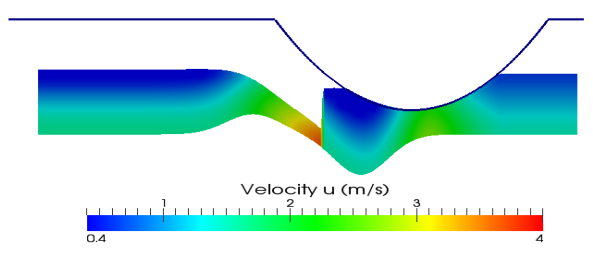

(a)

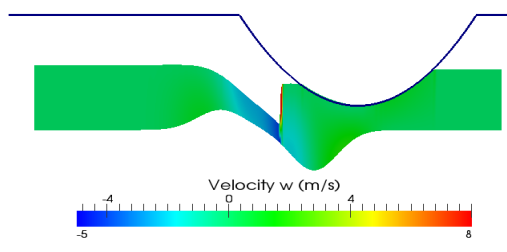

(b)

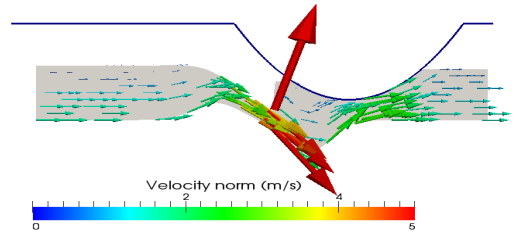

(c)

FIG. 5.3. Surface level of the solution with partially free surface (a) horizontal velocity $u$, (b) vertical velocity $w$, and (c) velocity norm (with arrows).

with $N=50, \alpha=3.5$, and $\beta=1$. The flow is fixed at inflow (left boundary) with $q_{i n}=\alpha$ and the water height is prescribed at outflow (right boundary) with $H_{\text {out }}=H_{0}\left(L_{x}\right)$. Following the results of paragraph 3.2, the water depth $H=H_{0}$ and the velocity fields $u, w$ given by (3.9), (3.10) are solutions of the considered problem. This situation where no shock occurs, is illustrated by figure 5.1.

5.2. Stationary solutions with a shock. Now we consider the situation described in paragraph 3.4. The bottom geometry is given by

$$
z_{b}(x)=0.2 e^{-0.5(x-8)^{2}}-0.4 e^{-0.5(x-12)^{2}}
$$




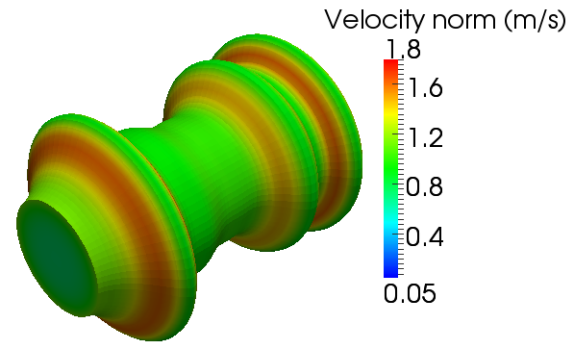

(a)

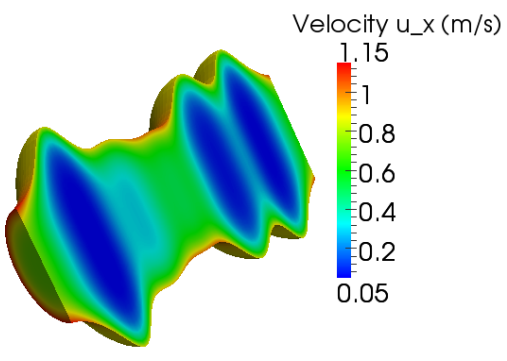

(b)

FIG. 5.4. Deformation of the cylinder and the velocity field (a) velocity norm, (b) slice along $z$ with the velocity $u_{x}$.

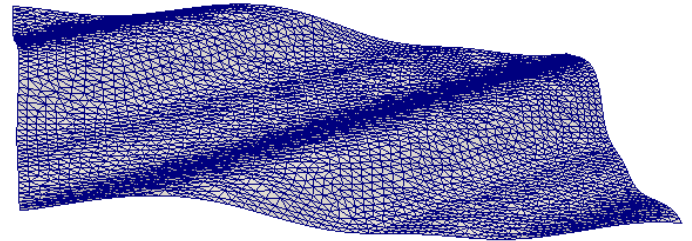

FIG. 5.5. 3d bottom geometry.

With this geometry and $\alpha=0.65, \beta=2.5$, and $H\left(L_{x}\right)=0.6$, the solution is discontinuous (cf. (3.19)) and governed by (3.20)-(3.21). The shock is characterized by (3.24)(3.26) and the vertical velocity $w$ is obtained from (3.10). The obtained solution is depicted in figure 5.2 .

\subsection{Interactions with structures.}

5.3.1. Analytical solutions with partially free surface. The analytical solutions proposed in paragraph 3.8.1 are illustrated here in figure 5.3.

We use the topography given by (5.1). As for figure 5.2, we choose $\alpha=0.65$ and $\beta=2.5$. The external pressure $p^{a}$ is given by (3.37) with $K(x)=100 x$ and $\eta_{m}(x)=$ $\min \left(1.2,0.2+0.04(x-14)^{2}\right)$.

5.3.2. Analytical solutions in cylindrical coordinates. The figure 5.4 depicts the analytical solution proposed in paragraph 3.8.2. It corresponds to a flow in a deformable cylinder of length $L_{x}=10 \mathrm{~m}$ with the parameters $\alpha=2.5, \beta=0.35$, and $\gamma=1$, and the functions

$$
R_{0}(x)=1+2 e^{-\left(x-\frac{L_{x}}{3}\right)^{2}}+e^{-\left(x-\frac{2 L_{x}}{3}\right)^{2}} \quad \text { and } \quad K(x)=1-0.6 \sin \left(\frac{\pi}{20}\left(x-\frac{L_{x}}{2}\right)^{2}\right) .
$$

5.4. Analytical solutions in 3d. In this paragraph we give an example of the $3 \mathrm{~d}$ analytic solutions obtained in Section 4.

In this $3 \mathrm{~d}$ situation, the water depth is given by

$$
H(x, y)=H_{0}\left(\alpha_{1} x+\alpha_{2} y\right)=1+0.3 \sin \left(\beta\left(\alpha_{1} x+\alpha_{2} y\right)\right),
$$




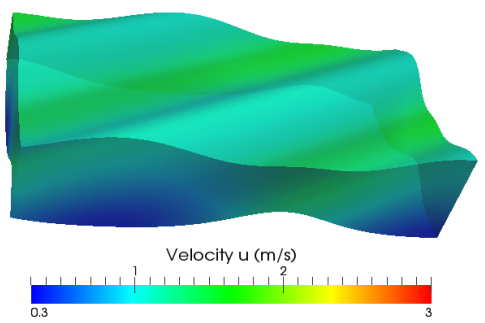

(a)

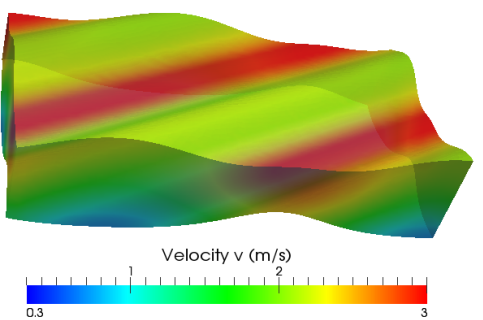

(b)

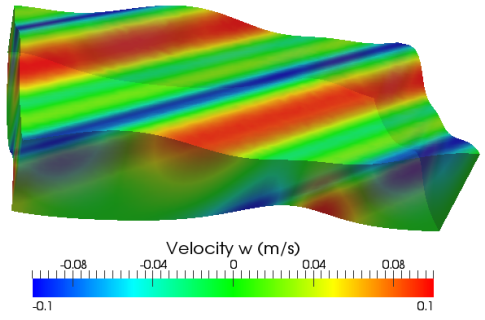

(c)

Fig. 5.6. Surface level of the $3 d$ solution and (a) and (b) horizontal velocities $u$ and $v$ and (c) vertical velocity $w$.

with $\alpha_{1}=1, \alpha_{2}=2$, and $\beta=1$. The bottom geometry is given by (4.10). The bottom profile with 3090 vertices and 5978 triangles is depicted in figure 5.5. The corresponding surface level and velocity fields $u, v$, and $w$ have been calculated from (4.5)-(4.7) and are presented in figure 5.6.

\section{Conclusion}

We have proposed analytical solutions for the $2 \mathrm{~d}$ and $3 \mathrm{~d}$ incompressible and hydrostatic Euler system. The FRESH-ASSESS solutions have been obtained in various situations: free surface, partially free surface, shocks, transient regime, et cetera, and can be derived for a large class of geometrical domains.

The main interest of these analytical solutions is to allow the validation of numerical schemes. The authors have already checked that the numerical schemes they have proposed $[1,2]$ for the resolution of the incompressible and hydrostatic Euler system with free surface are able to recover the analytical solutions proposed here.

Acknowledgment. The authors want to thank Emmanuel Audusse and Benoît Perthame for helpful discussions that have allowed us to greatly improve the paper.

\section{REFERENCES}

[1] E. Audusse, M.-O. Bristeau, M. Pelanti, and J. Sainte-Marie, Approximation of the hydrostatic Navier-Stokes system for density stratified flows by a multilayer model. Kinetic interpretation and numerical validation, J. Comp. Phys., 230, 3453-3478, 2011.

[2] E. Audusse, M.-O. Bristeau, B. Perthame, and J. Sainte-Marie, A multilayer Saint-Venant system with mass exchanges for shallow water flows. Derivation and numerical validation, ESAIM: M2AN, 45, 169-200, 2011.

[3] A.J.C. Barré de Saint-Venant, Théorie du mouvement non permanent des eaux avec applications aux crues des rivières et à l'introduction des marées dans leur lit, C.R. Acad. Sci. Paris, 73, 147-154, 1871. 
[4] P. Bonneton, E. Barthelemy, F. Chazel, R. Cienfuegos, D. Lannes, F. Marche, and M. Tissier, Recent advances in Serre-Green Naghdi modelling for wave transformation, breaking and runup processes, Euro. J. Mech. - B/Fluids, Special Issue: Nearshore Hydrodynamics, 30(6), 589-597, 2011.

[5] J.-V. Boussinesq, Théorie de l'intumescence liquide appelée onde solitaire ou de translation se propageant dans un canal rectangulaire, C. R. Acad. Sci. Paris, 72, 755-759, 1871.

[6] Y. Brenier, Homogeneous hydrostatic flows with convex velocity profiles, Nonlin., 12(3), 495$512,1999$.

[7] A.E. Green and P.M. Naghdi, A derivation of equations for wave propagation in water of variable depth, J. Fluid Mech., 78, 237-246, 1976.

[8] E. Grenier, On the derivation of homogeneous hydrostatic equations, ESAIM: M2AN, 33(5), 965-970, 1999.

[9] D.D. Houghton and A. Kasahara, Nonlinear shallow fluid flow over an isolated ridge, Commun. Pure Appl. Math., 21(1), 1-23, 1968.

[10] D.J. Korteweg and G. de Vries, On the change of form of long waves advancing in a rectangular canal, and on a new type of long stationary waves, Phil. Mag., 39, 422-443, 1895.

[11] M.D. Kruskal and N.J. Zabusky, Exact invariants for a class of nonlinear wave equations, J. Math. Phys., 7, 1256-1267, 1966.

[12] P.D. Lax, Integrals of nonlinear equations of evolution and solitary waves, Commun. Pure Appl. Math., 21(5), 467-490, 1968.

[13] T. Levi-Civita, Détermination rigoureuse des ondes permanentes d'ampleur finie, Math. Ann., 93(1), 264-314, 1925.

[14] I. MacDonald, M.J. Baines, N.K. Nichols, and P.G. Samuels, Comparisons of some Steady State Saint-Venant Solvers for some Test Problems with Analytic Solutions, Tech. Report 3/95, Dept. Math., University of Reading, 1995. http://www.reading.ac.uk/maths/research/maths-numanalrpts.aspx\#1995.

[15] I. MacDonald, M.J. Baines, N.K. Nichols, and P.G. Samuels, Steady Open Channel Test Problems with Analytic Solutions, Tech. Report 2/95, Dept. Math., University of Reading, 1995. http://www.reading.ac.uk/maths/research/maths-numanalrpts.aspx\#1995.

[16] A.J. Pullan, N.P. Smith, and P.J. Hunter, An anatomically based model of transient coronary blood flow in the heart, SIAM J. Appl. Math., 62(3), 990-1018, 2002.

[17] A. Ritter, Die fortpflanzung der wasserwellen, Vereine Deutscher Ingenieure Zeitswchrift (in German), 36(33), 947-954, 1892.

[18] J.J. Stoker, The formation of breakers and bores the theory of nonlinear wave propagation in shallow water and open channels, Commun. Pure Appl. Math., 1(1), 1-87, 1948.

[19] W.C. Thacker, Some exact solutions to the non-linear shallow-water wave equations, J. Fluid Mech., 107, 499-508, 1981. 